\title{
Correction to: Cross-reactive, natural IgG recognizing L. major promote parasite internalization by dendritic cells and promote protective immunity
}

\author{
Filiz Demircik ${ }^{1}$ Susanna Lopez Kostka ${ }^{2}$ - Stefan Tenzer ${ }^{3} \cdot$ Ari Waisman $^{1,4,5} \cdot$ Esther Von Stebut $^{2,6}$ (1)
}

Published online: 20 October 2021

○) Springer-Verlag GmbH Germany, part of Springer Nature 2021

Correction to: Journal of Molecular Medicine https://doi.org/10.1007/s00109-021-02137-4

The correct name of the $1^{\text {st }}$ Author is presented in this paper.

Publisher's Note Springer Nature remains neutral with regard to jurisdictional claims in published maps and institutional affiliations.

The original article can be found online at https://doi.org/10.1007/ s00109-021-02137-4.

Esther Von Stebut

esther.von-stebut@uk-koeln.de

1 Institute for Molecular Medicine, University Medical Center, Johannes Gutenberg University Mainz, Mainz, Germany

2 Department of Dermatology, University Medical Center, Johannes Gutenberg University Mainz, Mainz, Germany

3 Institute for Immunology, University Medical Center, Johannes Gutenberg University Mainz, Mainz, Germany

4 Research Center for Immunotherapy (FZI), University, Medical Center, Johannes Gutenberg University Mainz, Mainz, Germany

5 Focus Program in Translational Neuroscience (FTN), University Medical Center, Johannes Gutenberg University Mainz, Mainz, Germany

6 Department of Dermatology, Faculty of Medicine, University of Cologne, Cologne, Germany 